\title{
Discriminators of pseudoprogression and true progression in high-grade gliomas: A systematic review and meta-analysis
}

Christopher Taylor ( $\nabla$ chris.2.taylor@kcl.ac.uk)

King's College London

Justyna Ekert

Wellcome Centre for Human Neuroimaging

Viktoria Sefcikova

University College London

Naomi Fersht

University College London Hospitals NHS Foundation Trust

George Samandouras

National Hospital for Neurology and Neurosurgery

\section{Research Article}

Keywords: Pseudoprogression, Dynamic susceptibility contrast perfusion MRI, Dynamic contrastenhanced perfusion MRI, Diffusion-weighted imaging, High-grade glioma

Posted Date: February 4th, 2022

DOl: https://doi.org/10.21203/rs.3.rs-938779/v2

License: (c) (i) This work is licensed under a Creative Commons Attribution 4.0 International License. Read Full License 


\section{Abstract}

\section{Background}

High-grade gliomas remain the most common primary brain tumour with limited treatments options and early recurrence rates following adjuvant treatments. However, differentiating true tumour progression (TTP) from treatment-related effects or pseudoprogression (PsP), may critically influence subsequent management options. Structural MRI is routinely employed to evaluate treatment responses, but misdiagnosis of TTP or PsP may lead to continuation of ineffective or premature cessation of effective treatments, respectively.

\section{Methods}

A systematic review and meta-analysis were conducted in accordance with the Preferred Reporting Items for Systematic Reviews and Meta-analyses (PRISMA) method. The entirety of the Embase and MEDLINE databases were searched for methods differentiating PSP and TTP, and studies were selected using prespecified eligibility criteria. The sensitivity and specificity of included studies were summarised in a forest plot. Three of the identified methods were assessed in a separate subgroup meta-analysis.

\section{Results}

Twenty-six studies assessing seven distinct neuroimaging methods in 1145 patients were included in the systematic review. 18F-fluoroethyltyrosine PET (FET PET) and amide proton transfer-weighted MRI (APTw-MRI) showed high diagnostic accuracy, but results were based on single low-powered studies. The highest performing methods in the subgroup analysis were DWI (AUC $=0.95[0.92-0.96])$ and DSC-pMRI (AUC $=0.94[0.91-0.95])$, compared to DCE-pMRI (AUC $=0.90[0.87-0.93])$.

\section{Conclusions}

Both DWI and DSC-pMRI performed with high sensitivity and specificity for differentiating PsP from TTP. Considering the technical parameters and feasibility of each identified method, DSC-pMRI was found to be the most promising technique.

\section{Key Points}

- DSC-pMRI was identified as the most promising method for differentiating TTP from PSP

- DWI and DCE-pMRI also demonstrated high diagnostic accuracy respectively

- 18F-FET PET and APTW-MRI show promise but require further investigation

\section{Importance Of The Study}

Pseudoprogression is observed in approximately one-third of high-grade gliomas following radiotherapy. Histopathology, the gold standard for differentiating PsP from TTP, is invasive, time-consuming, and 
often not routinely employed to guide treatment following initial surgery. This study aims to compare imaging techniques differentiating PSP from TTP featuring high diagnostic accuracy and clinical feasibility, with the aim to identify those with the most potential for a more accurate and timelier clinical diagnosis, and providing clinical guidance for further treatment steps.

\section{Introduction}

High-grade gliomas (HGGs) remain some of the most common subtypes of primary brain tumours[1] with standard treatment options including surgical debulking followed by radiotherapy, and adjuvant or concurrent chemoradiation therapy (CCRT) with temozolomide, in world health organisation (WHO) grade III and IV, respectively.[2].[3] However, HGGs are characterised with early and high recurrence rates.[4] Routine MRI scans are often reported to display tumour volume growth, new or enlarged areas of contrast enhancement, or oedema which may represent either TTP or treatment effects called PsP. The latter can be transient and is often clinically asymptomatic,[5] but is often misdiagnosed as TTP, leading to premature cessation of potentially effective treatments and often substitution for less effective, secondline treatments.[6] Conversely, TTP misdiagnosed as PsP can complicate the monitoring of tumour progression by increasing waiting times, negatively influencing treatment outcomes.[7]

A recent meta-analysis by Abbasi and colleagues[8] found a form of pseudoprogression in $36 \%$ of 2603 patients harbouring HGGs. However, lack of standardised definitions for PsP and accurate diagnostic methods resulted in varying prevalence estimates in the literature. PsP occurs within the first 3 months following radiotherapy almost $60 \%$ of the time, with a range that is usually between 2 to 6 months.[9].[10] It is physiologically and clinically comparable to radiation necrosis, which generally occurs 3 to 12 months after therapy.[11] It is postulated that radiotherapy induces local endothelial cell death, leading to increased vascular permeability, perilesional oedema, and mass effect.[12] This gives a localised area of contrast enhancement on structural MRI that requires further assessment to distinguish from TTP.[12] Interestingly, patients with confirmed PsP generally have an improved prognosis,[13] although this may be subject to survivor bias due to the usually longer time PsP requires to manifest.[5]

Currently, standard practice involves clinical confirmation of PsP using serial MRI, histopathology via invasive brain biopsy, and/or application of the RANO criteria.[14] This practice is time-consuming and can be more subjective, and histological confirmation requires admission and additional surgery under general anaesthetic. Watchful surveillance can further delay clinical decision making, significantly affecting prognosis.[7] Several imaging methods allowing early differentiation between PsP and TTP, are currently under investigation and demonstrate high sensitivity and specificity compared to histological confirmation.

Numerous modalities have been reported including dynamic susceptibility contrast perfusion MRI (DSCpMRI), dynamic contrast-enhanced perfusion MRI (DCE-pMRI), diffusion-weighted MRI (DWI), arterial spin labelling (ASL), amide proton transfer-weighted MRI (APTw-MRI), 18F-fluoroethyltyrosine PET (FET-PET), magnetic resonance spectroscopy (MRS), and combinations of these modalities. All modalities have 
shown various degrees of diagnostic value, with no conclusive evidence of a superior technique. The aim of this systematic review and meta-analysis is to provide a comprehensive comparison of PsP and TTP differentiators based on measures of sensitivity, specificity, and clinical applicability. The advent of novel, more quantitative methods of diagnosis are also discussed.

\section{Methods}

Inclusion/Exclusion Criteria

Study design: Randomised controlled trials, controlled clinical trials, and prospective or retrospective observational studies were included with a sample size threshold $n \geq 10$. Only articles written in English were included. Conference abstracts and grey literature were excluded.

Population: Studies included adult patients ( $\geq 16$ years old) receiving radiotherapy or chemoradiotherapy following a diagnosis of a high-grade glioma. Studies needed to specify the proportion of the sample that exhibited PsP, which was confirmed no more than 6 months following radiotherapy.

Intervention: Studies assessed the diagnostic accuracy of a method used to differentiate TTP from PsP in HGG patients.

Outcome: Study outcomes reported a method's diagnostic sensitivity and specificity compared to the gold standard of expert histological examination and/or examination according the RANO criteria.

Search Strategy

Embase and MEDLINE were searched using Ovid on the 10th of November 2020 using Medical Subject Headings (MeSH) and text words related to iterations of the topics of HGGs and PsP. The search strategy (Supplementary Appendix A) was developed in consultation with a research librarian. Studies returned by the search strategy were compiled and screened for data extraction in accordance with the Preferred Reporting Items for Systematic Reviews and Meta-Analysis (PRISMA) method.[15] All titles and selected abstracts were screened on the basis of the inclusion criteria, and full texts were subsequently reviewed. The full selection process is shown in Figure 1, and a detailed list of exclusion criteria is provided in Supplementary Material C.

Data Extraction and Quality Assessment

Data were extracted onto a Microsoft Excel[16] spreadsheet with the following variables: (1) first author and year of publication; (2) neuroimaging method of discrimination between TTP and PSP; (3) sample size and demographics, including proportion of patients that presented with PsP; (4) administration of radiotherapy / chemoradiation therapy / CCRT; (5) tumour grade; and (6) sensitivity and specificity measures. Extracted data were inputted into Cochrane Review Manager 5.4.[17] The review was conducted according to the Meta-analysis of Observational Studies in Epidemiology (MOOSE) proposal. [18] The quality of included studies and risk of bias was assessed with the Quality Assessment of 
Diagnostic Accuracy (QUADAS-2) tool by two independent and blinded reviewers C.T. and V.S.[19] Any disagreement was resolved with consensus.

Data Synthesis and Statistical Analysis

All included studies were presented in a forest plot. A separate subgroup meta-analysis was performed to compare the three most prevalent methods among studies: DSC-pMRI $(n=11)$, DCE-pMRI $(n=4)$, and DWI $(n=9)$. For the systematic review, the primary outcomes were TTP and PsP discrimination method sensitivity and specificity. For the subgroup meta-analysis, the primary outcomes were pooled sensitivity, specificity, and area under the curve of the summary receiver operating characteristics curve (SROC AUC). [20]

Within-group heterogeneity was assessed using the $R^{2}$ variable, which describes the proportion of variation in study results that can be attributed to heterogeneity.[21] To account for the high heterogeneity of data across multiple modalities, data inputted into Cochrane Review Manager 5.4 were analysed using a random effects model, which assumes individual effects are uncorrelated with independent variables. Pooled parameters including sensitivity, specificity, likelihood ratio, negative likelihood ratio, diagnostic odds ratio (DOR) and SROC AUC were calculated using the MIDAS (meta-analytical integration of diagnostic test accuracy studies) package in STATA.[22] Forest plots and a subgroup analysis SROC plot were generated in Cochrane Review Manager 5.4. For studies reporting median instead of mean age, the mean was estimated according to the methods outlined by Hozo and colleagues.[23]

\section{Results}

Search Results

Following deduplication, the literature search yielded 1010 abstracts. Following abstract screening, 96 full-text articles were retrieved and assessed for eligibility. Data was extracted from 33 articles, seven of which did not report sufficient information to allow for calculation of sensitivity and specificity values, and therefore were excluded. A total of 26 studies totalling 1145 patients were included in the systematic review (see Table 1).

Of the 1145 patients, 453 (39.6\%) cases were confirmed to have PsP following radiotherapy, according to histological examination and/or examination according the RANO criteria. The mean age across the studies was 54, with a range of study mean age of 48 to 62 years. Based on 23 studies with relevant reporting, the male/female ratio was $1.8 / 1$.

Seven distinct methods for differentiating PsP from TTP were identified: DSC-pMRI, DWI, DCE-pMRI, ASL, APTw-MRI, 18F-FET PET, and conventional MRI. Two studies compared DSC-pMRI and DWI,[24],[25] one compared DSC-pMRI and ASL,[26] and one compared DSC-pMRI, ASL, and a combination of the two.[27] 
Data on seven distinct methods and two combinations of methods were included in the non-subgroup analysis (Figure 2A). The highest performing singular methods were FET PET (100\% sensitivity and $91 \%$ specificity) and APTw-MRI (95\% sensitivity and 92\% specificity), although these subgroups were based on single, low-powered studies.[28]'[29] Three studies (two using DSC-pMRI and one using DWI) reported $100 \%$ sensitivity and $100 \%$ specificity. The lowest sensitivity (38\%) for identifying tumour progression was reported by Young and colleagues,[30] who examined visual signs such as enhancement on conventional MRI across 93 patients. The lowest specificity for true progression (23\%) was reported by Kerkhof and colleagues,[31] which used visual interpretation of relative cerebral blood volume (rCBV) maps from DSC-pMRI.

Subgroup Analysis

Twenty-two studies assessing three distinct methods reported sufficient data to include in a separate set of subgroup analyses: DSC-pMRI $(n=11)$, DCE-pMRI $(n=4)$, and DWI $(n=9)$. Subgroups included 484, 186, and 309 patients, respectively. Studies by Kim and colleagues[24] and Prager and colleagues,[25] measured DSC-pMRI and DWI separately and thus, these studies appear twice in the forest plot (Figure 2B):

All three subgroups had a high diagnostic accuracy for differentiating PsP from TTP, supported by high diagnostic odds ratios (Figure 3, Table 2). The DWI subgroup showed the highest diagnostic accuracy (sensitivity $=0.91[0.84-0.95]$, specificity $=0.87$ [0.67 - 0.96], AUC $=0.95[0.92-0.96]$ ), followed by DSC-pMRI (sensitivity $=0.89[0.81-0.94]$, specificity $=0.89$ [0.68 -0.97$],$ AUC $=0.94[0.91-0.95]$ ). The sensitivity of DCE-pMRI and DWI to the identification of true tumour progression was higher than the specificity of both methods.

Heterogeneity for both DCE-pMRI and DWI was calculated as $R^{2}=0 \%$, but high heterogeneity was reported in the DSC-pMRI subgroup $\left(R^{2}=79 \%\right)$. This heterogeneity was more predominant in the reported specificity $\left(F^{2}=85 \%\right)$. The DSC-pMRI subgroup had the highest number of studies and the greatest variation in methodology. However, true heterogeneity is unlikely to be zero in the DCE-pMRI and DWI subgroups, and the small sample size may have led to an underestimation.[32]

\section{Quality Assessment}

Twelve of the included 26 studies were determined to have a high risk of bias. Nearly all included studies had a high risk of bias in the index test section. The parameter cut-off values were not pre-specified and instead defined post-hoc. High risk of bias was also apparent in patient selection. This was largely due to some studies including patients who received steroids with standard chemoradiotherapy. Details of patient enrolment and inclusion/exclusion were unclear in some studies, and nearly $40 \%$ of included patients presented with PSP, which is higher than previously reported.[8] There were low applicability concerns observed in the included studies. The full risk of bias table and a more detailed summary of quality assessment across all studies is detailed in Supplementary Material B1 \& B2. 


\section{Discussion}

The current systematic review and meta-analysis aimed to compare the most promising methods of the differentiation of PSP and TTP in patients with high-grade gliomas. A prior meta-analysis has compared the utility of DWI and PWI for discriminating PsP and TTP.[33] Consistent with our results, they did not find a significant difference between the two modalities. In contrast to the study by Tsakiris and colleagues, our inclusion criteria were not limited to two methods only. Furthermore, we consider each modality in the context of its clinical utility, aiming to provide a recommendation for physicians.

A total of seven PsP and TTP imaging discriminators have been identified: 1) DSC-pMRI, 2) DWI, 3) DCEpMRI, 4) ASL, 5) APTw-MRI, 6) 18F-FET PET, and 7) conventional MRI. Two combinations of the methods were included: 1) DSC-pMRI \& DCE-pMRI, and 2) DSC-pMRI \& ASL.

In the current review, the diagnostic accuracy measures, including the sensitivity and specificity for each method were extracted from the included studies and summarised in a forest plot. [34] Sensitivity was defined as the proportion of patients with histologically confirmed pseudoprogression out of all patients with suspected pseudoprogression based on neuroimaging results. Specificity is the ability of the test to identify cases of true tumour progression (and exclude pseudoprogression). It was defined as the proportion of patients with confirmed true tumour progression out of all patients that were deemed to have tumour progression based on neuroimaging findings. A high sensitivity and specificity constitute a high diagnostic accuracy, which in turn influences increases the overall precision of clinical decisions.

\section{Diffusion and Perfusion-based Methods}

Our meta-analysis found that DWI, DSC-pMRI and DCE-pMRI have high potential for differentiating PsP from TTP in patients harbouring HGGs. Out of these three methods, DWI demonstrated the highest sensitivity for detecting TTP $(0.91$ [0.84 - 0.95]), and DSC-pMRI demonstrated the highest specificity (0.89 [0.68 - 0.97]). A comparison of sensitivity and specificity for DWI, DSC-pMRI and DCE-pMRI did not reveal any statistically significant differences.

Assessment of imaging results using pre-specified parameter cut-off values was associated with higher sensitivity and specificity values in comparison to studies that relied on visual inspection. Kerkhof and colleagues[31] visually inspected rCBV colour maps to differentiate PsP from TTP, which yielded 72\% sensitivity and $23 \%$ specificity, both of which were the lowest values in the DSC-pMRI subgroup. In contrast, two other included DSC-pMRI studies[35][26] reported $100 \%$ sensitivity and $100 \%$ specificity using parameter cut-off values to differentiate PsP from TTP. Jovanovic and colleagues used a ratio of 2.89 of normalized CBV between the lesion and normal-appearing tissue, while Martínez-Martínez and colleagues used an rCBV value of 0.9 .

A direct comparison of DSC-pMRI and DWI was provided in studies by Kim and colleagues[24] and Prager and colleagues[25]. The former group found that the maximum CBV parameter of DSC-pMRI and the mean apparent diffusion coefficient (ADC) of DWI differentiated PSP from TTP with the same sensitivity 
(79\%) and specificity (45\%) in 34 patients. Prager and colleagues found DSC-pMRI to be $100 \%$ sensitive and $75 \%$ specific, although there were only four cases of PsP with available DSC-pMRI data. DWI performed with a sensitivity of $95 \%$ and specificity of $63 \%$ in the same study with eight PsP-confirmed patients.

Choi and colleagues[27] investigated the diagnostic accuracy of DSC-pMRI and ASL. The sensitivity and specificity of DSC-pMRI were determined as $82.4 \%$ and $67.9 \%$, respectively and $79.4 \%$ and $64.3 \%$, respectively, for ASL. A combination of the two modalities resulted in an increased sensitivity and specificity of $94.1 \%$ and $82.1 \%$, although this did not represent a significant increase in diagnostic accuracy $(p=0.133)$. Jovanovic and colleagues[26] separately assessed DSC-pMRI and ASL, and quantitative analysis found both methods yielded $100 \%$ sensitivity in their patient sample. For specificity, ASL scored $73 \%$ compared to $100 \%$ for DSC-pMRI.[26] All four included diffusion/perfusion-based methods show clinical potential. DSC-pMRI is currently the most widely employed, and its protocol and acquisition parameters are already well-defined.[36]

\section{FET PET}

There has been increasing interest in the application of PET in differentiating between PsP and TTP. One study included in this meta-analysis used 18F-FET PET and found the maximal tumour-to-brain ratio $\left(T_{B R}\right.$ max $)$ differentiates between the two with $100 \%$ specificity and $91 \%$ sensitivity at a cut-off of 2.3 , in a sample of 22 patients.[28] A similar cut-off value of $\mathrm{TBR}_{\max }=2.55$ was reported by Kebir and colleagues[37]. In the same study, a linear discriminant analysis-based algorithm was trained on IDHwildtype glioblastoma FET PET features and compared the results to a conventional FET PET analysis. [37] The algorithm provided an AUC of 0.93 , which was higher than the AUC for $\mathrm{TBR}_{\max }$ of 0.68 . Although this difference was significant when compared to other included parameters, it was not compared to $\operatorname{TBR}_{\max }(p=0.081)$.[37] This study was limited by a sample of 44 patients,[37] so further research with larger datasets would be necessary before considering clinical implementation.

\section{APTw-MRI}

APTw-MRI was used to differentiate PsP and TTP in one study only. Ma and colleagues[29] found APTwMRI to correctly identify 19 out of 20 patients in their TTP cohort (95\%) and 11 out of 12 patients in their PsP cohort (92\%). There was a marked signal increase in the TTP compared to PsP cohort, with an APTWmean cut-off of $2.42 \%$ and an APTWmax cut-off of $2.54 \%$.

\section{Combination Methods}

Multimodal approaches often demonstrate increased diagnostic accuracy and provide an additional layer of confidence compared to individual modalities. It is reasonable to assume the highest diagnostic accuracy would be achieved from the combination of results from already established modalities. However, the trade-off is the accompanied increase in cost and acquisition time. Regardless, with 
increasing availability of several above-mentioned modalities, the advantage of combination methods should be considered.

Two combination methods were included in the present review. A combination of $K^{\text {trans }}$ and rCBV maps obtained from DCE-pMRI and DSC-pMRI acquisitions, respectively, reported high sensitivity (88\%) and specificity (91\%) when applied to a cohort of 98 patients.[38] The maps could not discriminate between PsP and TTP in the cohort when used individually. Choi and colleagues[27] combined DSC-pMRI with ASL and reported sensitivity and specificity of $94 \%$ and $82 \%$, respectively, also finding the combination values higher than the individual methods. A prospective trial is currently registered to assess the diagnostic accuracy of DWI and ASL.[39]

Clinical Utility

Despite the large number of studies reporting the diagnostic potential of different imaging protocols, their routine clinical use has not been implemented. A summary of the main clinically relevant parameters is presented in Table 3. [40],[41]][42]

An inherent limitation of using perfusion-weighted imaging is that while perfusion parameters are generally lower in PsP, the associated inflammatory response is likely to influence perfusion and lead to increased perfusion parameters such as rCBV.[43] Similar effects have been seen with DWI as a result of radiation necrosis, suggesting decreased $A D C$ may not always reflect a high cellularity and TTP.[44] However, both PWI and DWI appear to demonstrate overall high diagnostic accuracy.

As the most frequently used perfusion MRI modality, DSC-pMRI may be preferable for standard protocol due to its high clinical availability and short acquisition time that can be under one minute.[42] The standardisation of rCBV discriminating cut-off values is limited by numerous potential imaging and data processing artifacts impeding accurate perfusion quantification as outlined by Willats and Calamante's 39 steps for accurate perfusion of DSC-pMRI data.[45] One of the most widely discussed issues is the possibility of contrast agent leakage into extracellular tissue, known as T1 shine-through effect [46]. Application of model-based leakage corrections is advised for single-echo gadolinium-based DSC-pMRI to account for the extent of vascular permeability.[47]

DCE-pMRI has a high signal-to-noise ratio compared to the other MR-perfusion techniques.[48] The main limitation of this method is the relatively long data acquisition time, often over several minutes. [49] Similar to other perfusion techniques, full quantification remains challenging due to difficulty in the DCE tracer modelling. Efforts are currently undertaken to resolve issues related to accurate quantification of perfusion techniques. The establishment of taskforces such as the Quantitative Imaging Biomarkers alliance will facilitate clinical implementation of methods by providing reference measures and guidelines for best practices [50].

ASL was a less frequently reported discriminating method compared to other perfusion methods. The main advantage of ASL over DSC-pMRI is that it does not require a gadolinium-based bolus injection. It 
may therefore be more suitable for patients with contraindications to administration of contrast agents. [51] Furthermore, ASL can acquire entirely quantitative values of cerebral blood flow (CBF). A nonsignificant increase in sensitivity and specificity was observed when CBF measures acquired using ASL was combined with DSC-pMRI, compared to use of the methods.[27] Jovanovic and colleagues[26] concluded that the diagnostic accuracy of ASL was sufficient to replace DSC-pMRI and therefore, avoid repeat follow-up contrast injections.[26] An important consideration of ASL is the longer acquisition time of 8-10 minutes at 1.5T and 4-5 minutes at 3T as well as significantly lower signal-to-noise ratio (SNR) compared to other perfusion methods.[42]

APTw is a novel imaging technique demonstrated to detect the increased mobile protein content in brain tumours[52]. Its full potential is yet to be established as U.S. Food and Drug Administration (FDA) approval of 3D-APTw for use on 3T clinical MRI scanners was granted in 2018.[53] However, APTw examinations may be time consuming ( 5-10 minutes) and are susceptible to magnetic field inhomogeneities.[54] Some recent work aims to optimise the signal-to-noise ratio[55] and image acquisition speed. APTw is a promising method with initial studies reporting a high diagnostic accuracy, but larger datasets are needed to compare its performance against other techniques.

Lastly, one study investigated the use of 18F-FET PET in diagnosing PSP.[28] Despite its high sensitivity and specificity, a long acquisition time of 50 minutes as reported by Galldiks and colleagues[28] limits clinical potential. Since 18F-FET PET relies on administration of labelled amino acid analogue, patients in the study were also required to fast for at least 12 hours before scanning. In contrast to other radiotracers, the half-life of fluorine-18 is long enough to allow for off-site production. The requirement for pharmacokinetic analysis with compartment modelling[56] further limits potential for clinical implementation.

\section{Future Directions}

Quantitative methods offer a more objective approach towards finding patterns in clinical data and enable more accurate diagnosis compared to qualitative methods.[57][58]

Jang and colleagues[59] recently applied a deep learning approach using convolutional neural networks to the differentiation of pseudoprogression and true progression, and achieved a sensitivity of $87 \%$ and a specificity of $94.5 \%$. Another study found a benefit of the combination of hypervascularity, cellularity and permeability parameters over single parameter measurements to distinguish the conditions.[60] The need for large datasets for training and testing radiomics models has led to a general lack of power, therefore future research should focus on increasing accessibility and data availability. National support for the scaling of technology and the potential use of artificial intelligence to aid clinical decision making has been outlined in the NHS Long Term Plan.[61]

\section{Conclusion}


Our systematic review and meta-analysis found DWI and DSC-pMRI have the highest diagnostic accuracy for differentiating between PsP and TTP. Considering the acquisition time and availability, DSC-pMRI holds high potential for clinical implementation. The risk of repeat contrast agent injections required for DSC-pMRI can be offset with the substitution of DSC-pMRI for ASL. There was a clear advantage of using parameter cut-offs, such as CBV over qualitative visual inspection. The diagnostic accuracy of methods such as PET, APTw-MRI, combination methods with MRS, and quantitative multiparametric techniques should be investigated in large-scale studies.

\section{Declarations}

Funding: The authors have no funding to report

Conflict of interest: The authors declare no conflict of interest

Availability of data and material: Not applicable

Code Availability: Not applicable

Ethics approval: Not applicable

Consent to participate: Informed consent was obtained from all individual participants included in the study.

Consent for publication: Informed consent was obtained from all individual participants included in the study.

\section{Acknowledgments}

The authors would like to thank UCL Queen Square deputy librarian Kate Brunskill for support in devising the search strategy of the systematic review.

\section{Author contributions}

All authors contributed to the study conception and design. The literature search was performed by Chris Taylor, and data analysis was also performed by Chris Taylor, with assistance from Justyna O. Ekert and Viktoria Sefcikova. The first draft of the manuscript was written by Chris Taylor and all authors commented on previous versions of the manuscript. Supervision and guidance were provided by George Samandouras and Naomi Fersht. All authors read and approved the final manuscript.

\section{References}

1. Lapointe S, Perry A, Butowski NA (2018) Primary brain tumours in adults. Lancet 392:432-446. https://doi.org/https://doi.org/10.1016/S0140-6736(18)30990-5 
2. Stupp R, Mason WP, van den Bent MJ, et al (2005) Radiotherapy plus Concomitant and Adjuvant Temozolomide for Glioblastoma. N Engl J Med 352:987-996.

https://doi.org/10.1056/nejmoa043330

3. Fernandes C, Costa A, Osório L, et al. (2017) Current Standards of Care in Glioblastoma Therapy. In: Glioblastoma. Codon Publications, pp 197-241

4. Birk HS, Han SJ, Butowski NA (2017) Treatment options for recurrent high-grade gliomas. CNS Oncol 6:61-70. https://doi.org/10.2217/cns-2016-0013

5. Thust SC, van den Bent MJ, Smits M. (2018) Pseudoprogression of brain tumors. J Magn Reson Imaging 48:571-589. https://doi.org/http://dx.doi.org/10.1002/jmri.26171

6. Ma Y, Wang Q, Dong Q, et al (2019) How to differentiate pseudoprogression from true progression in cancer patients treated with immunotherapy. Am J Cancer Res 9:1546-1553

7. Reichert A, Jacobs R (2018) The impact of waiting time on patient outcomes: Evidence from early intervention in psychosis services in England. Heal Econ (United Kingdom) 27:1772-1787. https://doi.org/10.1002/hec.3800

8. Abbasi AW, Westerlaan HE, Holtman GA, et al (2018) Incidence of Tumour Progression and Pseudoprogression in High-Grade Gliomas: a Systematic Review and Meta-Analysis. Clin Neuroradiol 28:401-411. https://doi.org/10.1007/s00062-017-0584-x

9. Zikou A, Sioka C, Alexiou GA, et al (2018) Radiation necrosis, pseudoprogression, pseudoresponse, and tumor recurrence: Imaging challenges for the evaluation of treated gliomas. Contrast Media Mol. Imaging 2018

10. Brandsma D, Stalpers L, Taal W, et al. (2008) Clinical features, mechanisms, and management of pseudoprogression in malignant gliomas. Lancet Oncol 9:453-461.

https://doi.org/http://dx.doi.org/10.1016/S1470-2045\%2808\%2970125-6

11. Fatterpekar GM, Galheigo D, Narayana A, et al (2012) Treatment-related change versus tumor recurrence in high-grade gliomas: A diagnostic conundrum - Use of dynamic susceptibility contrastenhanced (DSC) perfusion MRI. Am J Roentgenol 198:19-26.

https://doi.org/http://dx.doi.org/10.2214/AJR.11.7417

12. Ellingson BM, Chung C, Pope WB, et al (2017) Pseudoprogression, radionecrosis, inflammation or true tumor progression? challenges associated with glioblastoma response assessment in an evolving therapeutic landscape. J. Neurooncol. 134:495-504

13. Radbruch A, Fladt J, Kickingereder P, et al. (2015) Pseudoprogression in patients with glioblastoma: Clinical relevance despite low incidence. Neuro Oncol 17:151-159. https://doi.org/http://dx.doi.org/10.1093/neuonc/nou129

14. Chukwueke UN, Wen PY (2019) Use of the Response Assessment in Neuro-Oncology (RANO) criteria in clinical trials and clinical practice. CNS Oncol 8:CNS28-CNS28. https://doi.org/10.2217/cns2018-0007

15. Moher D, Shamseer L, Clarke M, et al (2015) Preferred reporting items for systematic review and meta-analysis protocols (PRISMA-P) 2015 statement. Syst Rev 4:1. https://doi.org/10.1186/2046- 
4053-4-1

16. Microsoft Corporation. Microsoft Excel 2016. Retrieved from https://office.microsoft.com/excel

17. (2020) Review Manager (RevMan) [Computer program]. Version 5.4, The Cochrane Collaboration, 2020.

18. Stroup DF, Berlin JA, Morton SC, et al (2000) Meta-analysis of observational studies in epidemiology: A proposal for reporting. J Am Med Assoc 283:2008-2012. https://doi.org/10.1001/jama.283.15.2008

19. Whiting PF, Rutjes AWS, Westwood ME, et al (2011) Quadas-2: A revised tool for the quality assessment of diagnostic accuracy studies. Ann. Intern. Med. 155:529-536

20. Jones CM, Athanasiou T (2005) Summary Receiver Operating Characteristic Curve Analysis Techniques in the Evaluation of Diagnostic Tests. Ann Thorac Surg 79:16-20. https://doi.org/10.1016/j.athoracsur.2004.09.040

21. Higgins JPT, Thompson SG (2002) Quantifying heterogeneity in a meta-analysis. Stat Med 21:15391558. https://doi.org/https://doi.org/10.1002/sim.1186

22. Dwamena B (2009) MIDAS: Stata module for meta-analytical integration of diagnostic test accuracy studies. Stat Softw Components

23. Hozo SP, Djulbegovic B, Hozo I (2005) Estimating the mean and variance from the median, range, and the size of a sample. BMC Med Res Methodol 5:13. https://doi.org/10.1186/1471-2288-5-13

24. Kim JYJH, Park JE, Jo Y, et al (2019) Incorporating diffusion- And perfusion-weighted MRI into a radiomics model improves diagnostic performance for pseudoprogression in glioblastoma patients. Neuro Oncol 21:404-414. https://doi.org/http://dx.doi.org/10.1093/neuonc/noy133

25. Prager AJ, Martinez N, Beal K, et al (2015) Diffusion and perfusion MRI to differentiate treatmentrelated changes including pseudoprogression from recurrent tumors in high-grade gliomas with histopathologic evidence. AJNR Am J Neuroradiol 36:877-885. https://doi.org/http://dx.doi.org/10.3174/ajnr.A4218

26. Jovanovic M, Radenkovic S, Stosic-Opincal T, et al. (2017) Differentiation between progression and pseudoprogression by arterial spin labeling MRI in patients with glioblastoma multiforme. J BUON 22:1061-1067

27. Choi YJ, Kim HS, Jahng G-H, et al (2013) Pseudoprogression in patients with glioblastoma: Added value of arterial spin labeling to dynamic susceptibility contrast perfusion MR imaging. Acta radiol 54:448-454. https://doi.org/http://dx.doi.org/10.1177/0284185112474916

28. Galldiks N, Dunkl V, Stoffels G, et al (2015) Diagnosis of pseudoprogression in patients with glioblastoma using 0-(2-[18F]fluoroethyl)-L-tyrosine PET. Eur J Nucl Med Mol Imaging 42:685-695. https://doi.org/10.1007/s00259-014-2959-4

29. Ma B, Blakeley JO, Hong X, et al. (2016) Applying amide proton transfer-weighted MRI to distinguish pseudoprogression from true progression in malignant gliomas. J Magn Reson Imaging 44:456462. https://doi.org/http://dx.doi.org/10.1002/jmri.25159 
30. Young RJ, Gupta A, G, Shah AD, et al. (2013) MRI perfusion in determining pseudoprogression in patients with glioblastoma. Clin Imaging 37:41-49.

https://doi.org/http://dx.doi.org/10.1016/j.clinimag.2012.02.016

31. Kerkhof M, Tans PL, Hagenbeek RE, et al (2017) Visual inspection of MR relative cerebral blood volume maps has limited value for distinguishing progression from pseudoprogression in glioblastoma multiforme patients. CNS Oncol 6:297-306. https://doi.org/10.2217/cns-2017-0013

32. von Hippel PT (2015) The heterogeneity statistic I(2) can be biased in small meta-analyses. BMC Med Res Methodol 15:35. https://doi.org/10.1186/s12874-015-0024-z

33. Tsakiris C, Siempis T, Alexiou GA, et al (2020) Differentiation Between True Tumor Progression of Glioblastoma and Pseudoprogression Using Diffusion-Weighted Imaging and Perfusion-Weighted Imaging: Systematic Review and Meta-analysis. World Neurosurg.

https://doi.org/10.1016/j.wneu.2020.07.218

34. Eusebi P (2013) Diagnostic accuracy measures. Cerebrovasc Dis 36:267-272. https://doi.org/10.1159/000353863

35. Martínez-Martínez A, Martínez-Bosch J. (2014) Perfusion magnetic resonance imaging for high grade astrocytomas: Can cerebral blood volume, peak height, and percentage of signal intensity recovery distinguish between progression and pseudoprogression? Radiologia 56:35-43. https://doi.org/http://dx.doi.org/10.1016/j.rx.2013.02.006

36. Welker K, Boxerman J, Kalnin A, et al (2015) ASFNR recommendations for clinical performance of MR dynamic susceptibility contrast perfusion imaging of the brain. AJNR Am J Neuroradiol 36:E41E51. https://doi.org/10.3174/ajnr.A4341

37. Kebir S, Schmidt T, Weber M, et al (2020) A preliminary study on machine learning-based evaluation of static and dynamic fet-pet for the detection of pseudoprogression in patients with idh-wildtype glioblastoma. Cancers (Basel) 12:1-14. https://doi.org/10.3390/cancers12113080

38. Elshafeey N, Kotrotsou A, Hassan A, et al. (2017) Multicenter study to demonstrate radiomic texture features derived from MR perfusion images of pseudoprogression compared to true progression in glioblastoma patients. J Clin Oncol 35:

39. Liu Z-C, Yan L-F, Hu Y-C, et al (2017) Combination of IVIM-DWI and 3D-ASL for differentiating true progression from pseudoprogression of Glioblastoma multiforme after concurrent chemoradiotherapy: study protocol of a prospective diagnostic trial. BMC Med Imaging 17:10. https://doi.org/10.1186/s12880-017-0183-y

40. Bammer R (2003) Basic principles of diffusion-weighted imaging. Eur J Radiol 45:169-184. https://doi.org/10.1016/S0720-048X(02)00303-0

41. Jahng G-H, Li K-L, Ostergaard L, Calamante F (2014) Perfusion magnetic resonance imaging: a comprehensive update on principles and techniques. Korean J Radiol 15:554-577. https://doi.org/10.3348/kjr.2014.15.5.554

42. Essig M, Shiroishi MS, Nguyen TB, et al (2013) Perfusion MRI: The five most frequently asked technical questions. Am. J. Roentgenol. 200:24-34 
43. Yoshii Y (2008) Pathological review of late cerebral radionecrosis. Brain Tumor Pathol. 25:51-58

44. Asao C, Korogi Y, Kitajima M, et al (2005) Diffusion-Weighted Imaging of Radiation-Induced Brain Injury for Differentiation from Tumor Recurrence. Am J Neuroradiol 26:

45. Willats L, Calamante F (2013) The 39 steps: evading error and deciphering the secrets for accurate dynamic susceptibility contrast MRI. NMR Biomed 26:913-931

46. Thompson G, Mills SJ, Coope DJ, et al (2011) Imaging biomarkers of angiogenesis and the microvascular environment in cerebral tumours. Br J Radiol 84:S127-S144

47. Boxerman JL, Schmainda KM, Weisskoff RM (2006) Relative cerebral blood volume maps corrected for contrast agent extravasation significantly correlate with glioma tumor grade, whereas uncorrected maps do not. Am. J. Neuroradiol. 27:859-867

48. Dietrich O, Biffar A, Baur-Melnyk A, Reiser MF (2010) Technical aspects of MR diffusion imaging of the body. Eur J Radiol 76:314-322. https://doi.org/10.1016/j.ejrad.2010.02.018

49. Chilla GS, Tan CH, Xu C, Poh CL (2015) Diffusion weighted magnetic resonance imaging and its recent trend-a survey. Quant Imaging Med Surg 5:407-422. https://doi.org/10.3978/j.issn.22234292.2015.03.01

50. McGehee BE, Pollock JM, Maldjian JA (2012) Brain perfusion imaging: how does it work and what should I use? J Magn Reson imaging 36:1257-1272

51. Gulani V, Calamante F, Shellock FG, et al (2017) Gadolinium deposition in the brain: summary of evidence and recommendations. Lancet Neurol 16:564-570.

https://doi.org/https://doi.org/10.1016/S1474-4422(17)30158-8

52. Isobe T, Matsumura A, Anno I, et al (2002) Quantification of cerebral metabolites in glioma patients with proton MR spectroscopy using T2 relaxation time correction. Magn Reson Imaging 20:343-349. https://doi.org/10.1016/S0730-725X(02)00500-3

53. U.S. Food and Drug Administration (2018) No

Titlehttps://www.accessdata.fda.gov/cdrh_docs/pdf17/K172920.pdf

54. Zhou J, Heo HY, Knutsson L, et al (2019) APT-weighted MRI: Techniques, current neuro applications, and challenging issues. J. Magn. Reson. Imaging 50:347-364

55. Zhou J (2011) Amide proton transfer imaging of the human brain. In: Magnetic Resonance Neuroimaging. Springer, pp 227-237

56. Debus C, Afshar-Oromieh A, Floca R, et al (2018) Feasibility and robustness of dynamic 18 F-FET PET based tracer kinetic models applied to patients with recurrent high-grade glioma prior to carbon ion irradiation. Sci Rep 8:1-17

57. Perry LA, Korfiatis P, Agrawal JP, et al. (2018) Increased signal intensity within glioblastoma resection cavities on fluid-attenuated inversion recovery imaging to detect early progressive disease in patients receiving radiotherapy with concomitant temozolomide therapy. Neuroradiology 60:35-42. https://doi.org/http://dx.doi.org/10.1007/s00234-017-1941-9 
58. Sollmann N, Gutbrod-Fernandez M, Burian E, et al. (2020) Subtraction maps derived from longitudinal magnetic resonance imaging in patients with glioma facilitate early detection of tumor progression. Cancers (Basel) 12:1-14. https://doi.org/http://dx.doi.org/10.3390/cancers12113111

59. Jang B-S, Park AJ, Jeon SH, et al (2020) Machine Learning Model to Predict Pseudoprogression Versus Progression in Glioblastoma Using MRI: A Multi-Institutional Study (KROG 18-07). Cancers (Basel) 12:. https://doi.org/10.3390/cancers12092706

60. Park JE, Kim HS, Goh MJ, et al (2015) Pseudoprogression in Patients with Glioblastoma: Assessment by Using Volume-weighted Voxel-based Multiparametric Clustering of MR Imaging Data in an Independent Test Set. Radiology 275:792-802. https://doi.org/10.1148/radiol.14141414

61. NHS. The NHS Long Term Plan. Published 2019. Accessed July 1, 2021. https://www.longtermplan.nhs.uk/wp-content/uploads/2019/01/nhs-long-term-plan-june-2019.pdf

62. Baek HJ, Kim HS, Kim N, et al. (2012) Percent change of perfusion skewness and kurtosis: A potential imaging biomarker for early treatment response in patients with newly diagnosed glioblastomas. Radiology 264:834-843. https://doi.org/http://dx.doi.org/10.1148/radiol.12112120

63. Cha J, Kim ST, Kim H-J, et al (2014) Differentiation of tumor progression from pseudoprogression in patients with posttreatment glioblastoma using multiparametric histogram analysis. AJNR Am J Neuroradiol 35:1309-1317. https://doi.org/10.3174/ajnr.A3876

64. Kong D-S, Kim ST, Kim E-H, et al (2011) Diagnostic dilemma of pseudoprogression in the treatment of newly diagnosed glioblastomas: the role of assessing relative cerebral blood flow volume and oxygen-6-methylguanine-DNA methyltransferase promoter methylation status. AJNR Am J Neuroradiol 32:382-387. https://doi.org/10.3174/ajnr.A2286

65. Mangla R, Singh G, Ziegelitz D, et al (2010) Changes in relative cerebral blood volume 1 month after radiation-temozolomide therapy can help predict overall survival in patients with glioblastoma. Radiology 256:575-584. https://doi.org/10.1148/radiol.10091440

66. Mihailović J, Daković M (2019) Advanced magnetic resonance techniques in early differentiation of pseudoprogression versus progression in the patients with glioblastoma multiforme. Vojnosanit Pregl 76:510-517. https://doi.org/10.2298/VSP170114108M

67. Bulik M, Kazda T, Slampa P, Jancalek R (2015) The Diagnostic Ability of Follow-Up Imaging Biomarkers after Treatment of Glioblastoma in the Temozolomide Era: Implications from Proton MR Spectroscopy and Apparent Diffusion Coefficient Mapping. Biomed Res Int 2015:641023. https://doi.org/10.1155/2015/641023

68. Chu HH, Choi SH, Ryoo I, et al (2013) Differentiation of true progression from pseudoprogression in glioblastoma treated with radiation therapy and concomitant temozolomide: comparison study of standard and high-b-value diffusion-weighted imaging. Radiology 269:831-840. https://doi.org/10.1148/radiol.13122024

69. Kazda T, Bulik M, Pospisil P, et al. (2016) Advanced MRI increases the diagnostic accuracy of recurrent glioblastoma: Single institution thresholds and validation of MR spectroscopy and 
diffusion weighted MR imaging. Neurolmage Clin 11:316-321.

https://doi.org/http://dx.doi.org/10.1016/j.nicl.2016.02.016

70. Lee WJ, Choi SH, Park C-K, et al (2012) Diffusion-weighted MR Imaging for the Differentiation of True Progression from Pseudoprogression Following Concomitant Radiotherapy with Temozolomide in Patients with Newly Diagnosed High-grade Gliomas. Acad Radiol 19:1353-1361. https://doi.org/http://dx.doi.org/10.1016/j.acra.2012.06.011

71. Reimer C, Deike K, Graf M, et al (2017) Differentiation of pseudoprogression and real progression in glioblastoma using ADC parametric response maps. PLoS One 12:e0174620. https://doi.org/10.1371/journal.pone.0174620

72. Song YS, Choi SH, Park C-K, et al (2013) True progression versus pseudoprogression in the treatment of glioblastomas: a comparison study of normalized cerebral blood volume and apparent diffusion coefficient by histogram analysis. Korean J Radiol 14:662-672.

https://doi.org/10.3348/kjr.2013.14.4.662

73. Yoo RE, Choi SH, Kim TM, et al. (2015) Independent poor prognostic factors for true progression after radiation therapy and concomitant temozolomide in patients with glioblastoma: Subependymal enhancement and low adc value. Am J Neuroradiol 36:1846-1852. https://doi.org/http://dx.doi.org/10.3174/ajnr.A4401

74. Nam JG, Kang KM, Choi SH, et al. (2017) Comparison between the prebolus T1 measurement and the fixed T1 value in dynamic contrast-enhanced MR imaging for the differentiation of true progression from pseudoprogression in glioblastoma treated with concurrent radiation therapy and temozolomide che. Am J Neuroradiol 38:2243-2250. https://doi.org/http://dx.doi.org/10.3174/ajnr.A5417

75. Suh $\mathrm{CH}$, Kim HS, Choi YJ, et al (2013) Prediction of pseudoprogression in patients with glioblastomas using the initial and final area under the curves ratio derived from dynamic contrastenhanced T1-weighted perfusion MR imaging. Am J Neuroradiol 34:2278-2286. https://doi.org/http://dx.doi.org/10.3174/ajnr.A3634

76. Thomas AA, Arevalo-Perez J, Kaley T, et al (2015) Dynamic contrast enhanced T1 MRI perfusion differentiates pseudoprogression from recurrent glioblastoma. J Neurooncol 125:183-190. https://doi.org/http://dx.doi.org/10.1007/s11060-015-1893-z

77. Yun TJ, Park CK, Kim TM, et al. (2014) Glioblastoma treated with concurrent radiation therapy and temozolomide chemotherapy: Differentiation of true progression from pseudoprogression with quantitative dynamic contrast-enhanced MR Imaging. Radiology 274:830-840. https://doi.org/http://dx.doi.org/10.1148/radiol.14132632

\section{Tables}

Tables 1-3 are not available with this version.

\section{Figures}


Figure 1

Legend not included with this version.

Figure 2

Legend not included with this version.

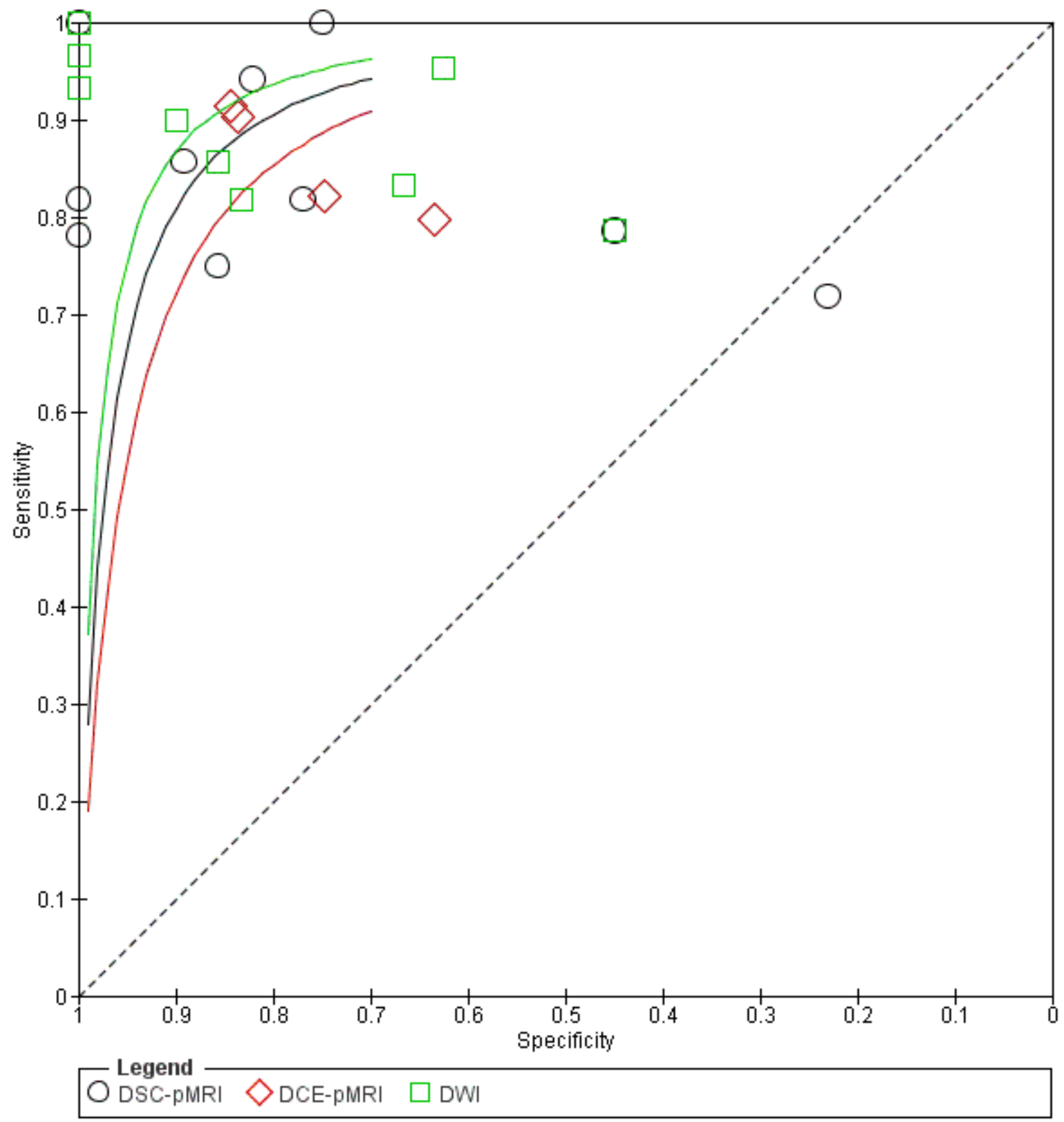

Figure 3 
Legend not included with this version.

\section{Supplementary Files}

This is a list of supplementary files associated with this preprint. Click to download.

- CTaylorSupplementary0921.docx 